\title{
CMIB: Unsupervised Image Object Categorization in Multiple Visual Contexts
}

\author{
Xiaoqiang Yan, Yangdong Ye, Hui Yu, Milos Manic
}

\begin{abstract}
Object categorization in images is fundamental to various industrial areas, such as automated visual inspection, fast image retrieval and intelligent surveillance. Most existing methods treat visual features (e.g., scale-invariant feature transform, SIFT) as content information of the objects, while regarding image tags as its contextual information. However, the image tags can hardly been acquired in complete unsupervised settings, especially when the image volume is too large to be marked. In this work, we propose a novel and effective method called contextual multivariate information bottleneck (CMIB) to discover object category in totally unlabeled images. Unlike treating image tags as the object's context, CMIB adopts one feature representation of the images to characterize the object's content information, while regarding the auxiliary clusterings obtained by other multiple related features as its visual contexts. In the proposed CMIB framework, we borrow the idea of the data compression procedure for object category discovery, which aims to squeeze the source image collection into its compressed representation as much as possible, while maximally preserving the correlative information between the content and visual contexts. Specifically, two Bayesian networks are built to characterize the relationships between data compression and information preservation. Moreover, a sequential informationtheoretic optimization is proposed to ensure the convergence of the CMIB objective function. Extensive experiments on five real-world image data sets show that the proposed method can significantly outperform the state-of-the-art baselines.
\end{abstract}

Index Terms-Object category discovery, visual context, information bottleneck, mutual information, Bayesian networks.

\section{INTRODUCTION}

$\mathbf{O}$ BJECT categorization in images has been an active and fundamental research topic in various industrial areas, such as automated visual inspection [1], fast image retrieval [2] and intelligent surveillance [3]. Recently, contextual information, a sort of available and complementary information that provides rich positive details for target data, can enhance the accuracy of object categorization models. In realistic images, many meaningful factors can be seen as the object's context, such as the tags of the target object, the scene where the object is performed in, and the related objects it played with.

At present, contextual information for supervised object categorization has been fully explored [4], [5], [6], [7], [8], in which the artificial marks are usually treated as the contexts of the target object, such as image tags, category label, descriptive captions. However, supervised object categorization methods with artificial marks have several apparent limitations. Firstly, supervised methods need sufficient ground-truth labels to train the classifiers, which is a labor intensive procedure and impractical for many industrial applications. Secondly, the artificial contexts (e.g., image tags, category label) must be obtained with the assistance of the priori knowledge of human experts, which limits its application scope, especially when the data volume is too large to be marked.

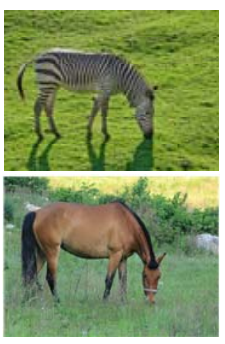

(a) Zebra \& Horse

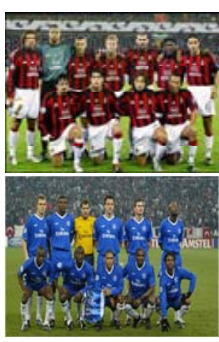

(b) Soccer Team

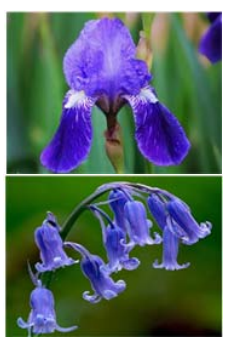

(c) Iris \& Bluebell

\begin{tabular}{c|c|c|c} 
& Zebra \& Horse & Soccer Team & Iris \& Bluebell \\
\hline Shape & $X$ & $X$ & \\
\hline Color & $\searrow$ & $\checkmark$ & $X$ \\
\hline Texture & $\searrow$ & $X$ &
\end{tabular}

Fig. 1. Above: three groups of similar objects from real-world images, which are (a) zebra and horse, (b) soccer teams, (c) iris and bluebell flower. Below: an example illustration of the object/contexts relationship. We can observe that it is hardly to differentiate objects just according to single content information. (Best viewed in color)

To overcome the limitations of the methods based on manual marks, several research works [9], [10], [11], [12] have been dedicated to exploring the object's contextual information in unsupervised settings, which utilize multiple visual features to characterize the content and context information of the images. However, the existing unsupervised methods always treat the content and contextual information equivalently, which may be problematic in realistic applications. Besides, due to the imaging variations (e.g., cluttered background, viewpoint changes), the content and contextual information of the target object always have different structures, and their feature distributions are also heterogeneous to each other. Therefore, integrating the heterogeneous content and contextual information together inappropriately will degrade the performance of the unsupervised object categorization models. More importantly, the visual features are usually represented by several high dimensional descriptors, dealing with them simultaneously always results in the problem of dimensionality curse.

In this study, we propose a novel contextual multivariate information bottleneck (CMIB) method to discover the hidden object category in unlabeled image collections. Opposite to previous works, CMIB adopts one feature representation of the object as its content information, while treating the auxiliary clusterings obtained by other multiple related features as its visual contexts. The visual contexts are discriminative enough to differentiate the objects in the unlabeled images. 
For instance, as shown in Fig. 1, it is hardly to differentiate the zebra and horse just using shape information. However, if we regard color and texture feature as visual contexts, the zebra and horse can be distinguished easily. In the framework of CMIB, the object category discovery is regarded as data compression through a "bottleneck", in which the images with similar objects are squeezed into together by maximally maintaining the information with respect to both content and visual contexts. Specifically, two Bayesian networks are constructed to characterize the relationships between the data compression and information preservation, in which multiinformation is built to measure the similarity of content and visual context. Moreover, a sequential information-theoretic solution is proposed to ensure the convergence of the objective function of CMIB. Our method outperforms the state-of-theart baselines on five real-world image data sets. To summarize, the main contributions of this work are:

- We propose a novel unsupervised object categorization method called CMIB, which can discover the object category in unlabeled images by considering its content and multiple visual contexts simultaneously.

- To the best of our knowledge, this is the first work to define the auxiliary clusterings as the object's visual context, which avoids the problem of dimensionality curse when dealing with multiple visual features simultaneously. We believe this basic idea can be generalized to many other related fields.

- We construct two Bayesian networks to characterize the relationships between the content and visual contexts. Besides, a sequential information-theoretic optimization solution is proposed to ensure the convergence of the objective function of CMIB.

- The proposed method is totally unsupervised and outperforms the existing state-of-the-art baselines on several benchmark data sets.

This paper is structured as follows. Section II analyzes the related work about context-based clustering methods and the background of MIB. Section III formulates the proposed CMIB. In Section IV, we report and discuss the experimental results. Section V concludes the paper finally.

\section{RELATED WORK}

\section{A. Unsupervised Context Based Methods}

In recent years, context based clustering [9], [10], [13], [11], [14], [12] has been a valuable unsupervised learning topic in machine learning and its various industrial applications. However, all the existing context based clustering approaches treat the target object's content and contextual information equivalently. In practice, the content and contextual information of same object have its own structures, and the distributions of them are always heterogeneous to each other. Thus, inappropriately integrating them together will degrade the clustering performance.

Essentially, contextual information is a complement to the content information of an object. In this regard, it would be pertinent to discuss multi-view and ensemble clustering methods. Both of them aim to improve the performance of

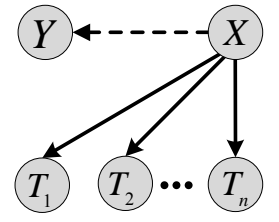

(a) $\mathcal{I}^{G_{m}}$ Data compression

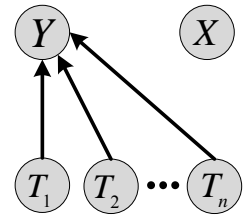

(b) $\mathcal{I}^{G_{\text {out }}}$ Information preservation
Fig. 2. Structural representation of $G_{i n}$ and $G_{o u t}$ in MIB framework. (a) In data compression part, the solid arrows from $X$ to $T_{1}, \cdots, T_{n}$ represent to find multiple compressed representations of $X$, while the dashed arrow between $X$ and $Y$ means that there exists a joint distribution $p(X, Y)$. (b) In information preservation part, the solid arrows denote the information that should be maximized with respect to $Y . \mathcal{I}^{G_{\text {in }}}$ and $\mathcal{I}^{G_{\text {out }}}$ are the amount of information in these two networks.

unsupervised object categorization model by considering the complementary effect of multiple related components. Specifically, multi-view clustering methods [15], [16], [17] aims to construct mappings, connections or agreements between multiple distinct views of the objects. In multi-view methods, the complementary views can be seen as the contexts of other views. In particular, local visual features are the most prevalent way to represent the different views of the objects in images. However, the dimensions of these visual features are always very high especially when dealing with them simultaneously. Moreover, how to control the balance of different views also remains a challenging task.

Different from multi-view clustering methods, ensemble clustering approaches [18], [19] refer to combining different clusterings of a given data collection into a single partition that is a better fit than existing clusterings. The information provided by auxiliary clusterings can also be seen as the context of target data. However, the existing ensemble clustering methods yield the final partition without accessing to the original feature representations of the images, which limits the final results in terms of the quality of existing base clusterings. In this study, we intend to discover the object category in unlabeled images by considering its content (local feature) and visual contexts (auxiliary clusterings) simultaneously.

\section{B. Multivariate information bottleneck}

The information bottleneck (IB) method [20] is an important branch of information theory, which aims to summarize a source data collection $X$ with its relevant term $Y$. Multivariate information bottleneck (MIB) [21] is an extension of the original IB, which uses the concept of multi-information to quantify the shared information between more than two variables. Given several variables $X_{1}, X_{2}, \ldots, X_{n}$, the multiinformation between these variables is defined as follows

$\mathcal{I}\left(X_{1}, \cdots, X_{n}\right)=D_{K L}\left[p\left(x_{1}, \cdots, x_{n}\right) \| p\left(x_{1}\right) \cdots p\left(x_{n}\right)\right]$.

In MIB framework, Bayesian network is adopted to characterize the relationships of multiple variables. Given a set of random variables $\mathbf{X}=\left\{X_{1}, \cdots, X_{n}\right\}$ and a set of compressed variables $\mathbf{T}=\left\{T_{1}, \cdots, T_{n}\right\}$, a Bayesian network is a graph in which vertices are the names of random variables (see Fig. 2). In particular, MIB uses Baysian networks $G_{i n}$ and $G_{\text {out }}$ to specify relations among variables. Both $G_{\text {in }}$ and $G_{\text {out }}$ 
are defined over $\mathbf{X} \bigcup \mathbf{T}$, and $G_{i n}$ indicates which subset of $\mathbf{X}$ is compressed into $\mathbf{T}, G_{\text {out }}$ specifies which relevant information should be preserved with respect to $Y$. The MIB functional can be written as

$$
\mathcal{L}_{\text {min }}=\mathcal{I}^{G_{\text {in }}}(\mathbf{X}, \mathbf{T})-\beta \cdot \mathcal{I}^{G_{\text {out }}}(\mathbf{X}, \mathbf{T}),
$$

where $\beta$ strikes a balance between the data compression information preservation in $G_{\text {in }}$ and $G_{\text {out }}$.

\section{CONTEXTUAL MUlTivariate INFORMATION BOTTLENECK}

Most existing object categorization methods treat visual features (e.g., scale-invariant feature transform, SIFT) as content information of the objects, while regarding artificial marks as its contextual information. However, the artificial marks can hardly been acquired in complete unsupervised settings, especially when the image volume is too large to be marked. In this paper, we propose a novel unsupervised object categorization method called CMIB, which can discover the object category in unlabeled images by considering its content and visual contexts simultaneously.

\section{A. Problem definition}

Local features are widely used in a variety of contentbased vision tasks [22], [23], [24]. For the task of object categorization, there are also various effective feature extraction techniques for characterizing the objects in images, such as scale-invariant feature transform (SIFT) [25], Color Attention [26], local binary patterns (LBP) [27]. In this paper, we treat one visual feature of the target object as its content information, while regarding the auxiliary clusterings obtained by other multiple related features as its visual contexts. First, we give the definition of visual contexts.

Definition 1. Suppose there are $k+1(k \geq 1)$ feature representations of the object in image collection $X$, if one of them is adopted as content information $Y$, then the visual contexts of the object is the clusterings $W_{1}, W_{2}, \cdots, W_{k}$ generated by the other $k$ remaining features.

To discover object categories, we consider an unlabeled image collection $X$, in which there is a set of samples $\left\{x_{1}, x_{2}, \cdots, x_{n}\right\}$ including various objects. Each data sample in the collection can be characterized by $k+1(k \geq 1)$ features, such as SIFT, TPLBP, Color Attention. Correspondingly, we can construct $k+1$ joint distributions for all the features according to the prevalent bag-of-visual-words (BoVW) model [28]. First, one discriminative feature is designated as the content information $Y=\left\{y_{1}, y_{2}, \cdots, y_{N}\right\}$, where $N$ is the codebook size of the BoVW model. Then, any clustering algorithms with promising performance (e.g., IB algorithm in this study) can be utilized to construct multiple basic clusterings $W_{1}, W_{2}, \cdots, W_{k}$ according to the other remaining $k$ features, which are regarded as visual contexts of the target objects. Our main goal is to find the potential object categories $T$ hidden in the unlabeled image collection $X$. In other words, the task of CMIB is to find an optimal encoding scheme $p(t \mid x)$ from $X$ to $T$, while maintaining the preservation of the content and visual contexts maximally.

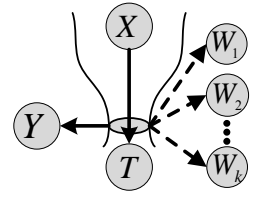

(a) $\mathcal{I}^{G_{i m}}$ Data compression

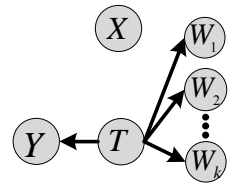

(b) $\mathcal{I}^{G_{o u}}$ Information preservation
Fig. 3. The model of CMIB method for unsupervised object category discovery in images. (a) In this part, the solid arrow from $X$ to $T$ means $X$ is mapped into its compressed representation $T$. At the same time, the solid and dashed arrows from the bottleneck to $Y$ and $W_{1}, W_{2}, \cdots, W_{k}$ mean that there are one content variable $Y$ and multiple contextual variables respectively. (b) In this part, the solid arrows specify what information should be maximized about content information $Y$ and visual contexts $W_{1}, W_{2}, \cdots, W_{k}$.

\section{B. Objective Function of CMIB}

In this part, the objective function of CMIB algorithm is presented. CMIB treats one feature variable of the objec$\mathrm{t}$ as the content information $Y$, while exploiting the base clusterings $W_{1}, W_{2}, \cdots, W_{k}$ obtained by other features as its visual contexts. As illustrated in Fig. 3. CMIB involves two parts: data compression and information preservation. We construct two Bayesian networks $G_{i n}$ and $G_{\text {out }}$ to characterize the relationships between the variables in the two parts. In particular, the network $G_{i n}$ is on behalf of the compressing relationship from $X$ to $T$, while $G_{\text {out }}$ expresses that the compressed variable $T$ should preserve the relevant information of content information $Y$ and visual context $W_{1}, W_{2}, \cdots, W_{k}$ simultaneously. Similar to [21], the multiinformation in $G_{\text {in }}$ and $G_{\text {out }}$ can be defined as follows

$$
\begin{aligned}
& \mathcal{I}^{G_{i n}}=I(X ; T), \\
\mathcal{I}^{G_{\text {out }}}= & I(T ; Y)+\sum_{i=1}^{k} I\left(T ; W_{i}\right),
\end{aligned}
$$

where $I(p ; q)$ is the term of mutual information [29] measuring the shared information between $p$ and $q . I(X ; T)$ measures how many bits that conveyed from the source variable $X$ to its compressed representation $T . I(T ; Y)$ measures how much information the variable $T$ maintains about the content information $Y$, and $\sum_{i=1}^{k} I\left(T ; W_{i}\right)$ measures the information contained in variable $T$ about the visual contexts $W_{1}, W_{2}, \cdots, W_{k}$ respectively. Thus, the objective function of CMIB can be written as follows according to Eq. (2)

$$
\begin{aligned}
& \mathcal{L}_{\text {max }}\{p(t \mid x)\}=\mathcal{I}^{G_{\text {out }}}-\beta^{-1} \cdot \mathcal{I}^{G_{\text {in }}} \\
& =I(T ; Y)+\sum_{i=1}^{k} I\left(T ; W_{i}\right)-\beta^{-1} I(X ; T),
\end{aligned}
$$

where $\beta$ strikes a balance between information preservation and data compression.

Now, the remaining task of the unsupervised object categorization is to maximize the value of objective function (5). To ensure its convergence, we present a sequential informationtheoretic solution, which always performs better than agglomerative methods. In this work, we concentrate on the hard clustering setting, where the value of $p(t \mid x)$ is 0 or 1 . Now, the task of CMIB becomes to find the hidden object categories in unlabeled image collection $X$ by maximizing the preservation of the information in objective function (5). 


\section{Optimization of $C M I B$}

To solve the problem of maximizing the objective function (5), we propose a sequential information-theoretic optimization, which is a "draw-and-merge" procedure essentially. Firstly, CMIB partitions the source image collection $X$ into $M$ categories stochastically. Then at the following iterative step, each single image $x \in X$ is "drawn" from its original category and is treated as a new category $\{x\}$. Now we should merge $\{x\}$ into category $t^{\text {new }}$ that causes the information loss minimization. That is, the optimization approach ought to increase the value of function (5) of CMIB algorithm. Let $\Delta \mathcal{L}$ be the value change after each draw and merge procedure, which is also called "merger cost". To maximize the value of Eq. (5), the single category $\{x\}$ should be merged into the category $t^{\text {new }}$ such that $t^{\text {new }}=\arg \min _{t \in T} \Delta \mathcal{L}$, where $\Delta \mathcal{L}$ indicates the information loss of the Eq. (5) after merging $\{x\}$ into the category $t$.

Definition 2. Suppose a certain $\{x\}$ be merged into some category $t$, and thus generate another new category $t^{\prime}$, the probability change caused by the merge step is defined as

$$
\left\{\begin{array}{l}
p\left(t^{\prime}\right)=p(x)+p(t), \\
p\left(y \mid t^{\prime}\right)=\frac{p(x)}{p\left(t^{\prime}\right)} p(y \mid x)+\frac{p(t)}{p\left(t^{\prime}\right)} p(y \mid t),
\end{array}\right.
$$

where $p\left(t^{\prime}\right)$ is the prior probability after merging $\{x\}$ into category $t, p\left(y \mid t^{\prime}\right)$ is the joint probability distribution of category $t^{\prime}$ over the feature variable $Y$.

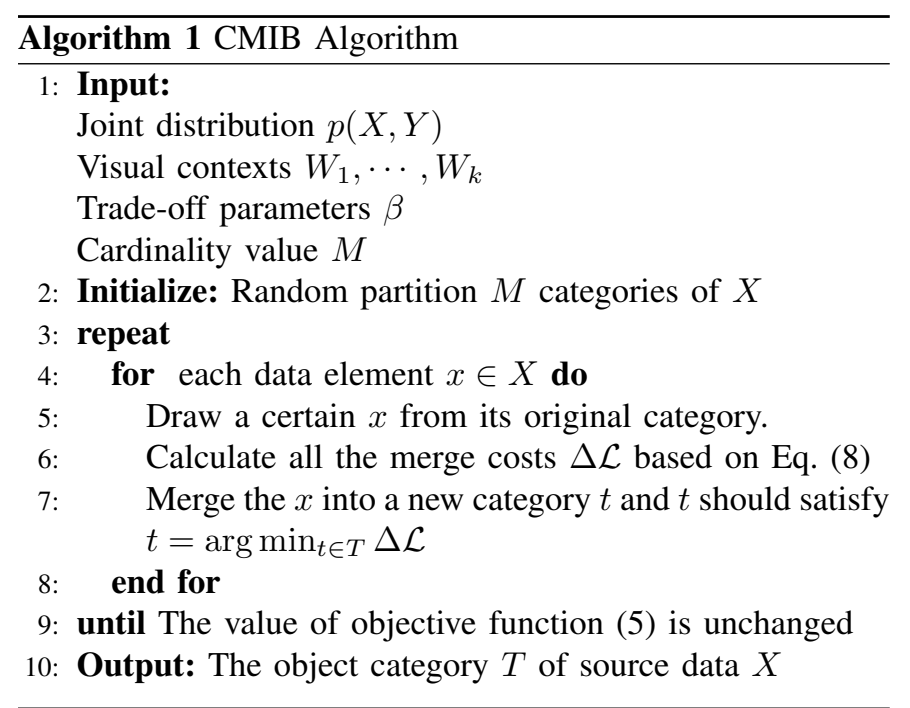

Let $\mathcal{L}^{\text {bef }}$ and $\mathcal{L}^{\text {new }}$ stand for the value of the Eq. (5) before and after the single category $x$ is merged into the category $t$. Then the merge cost $\Delta \mathcal{L}$ can be calculated as follows

$$
\begin{aligned}
& \Delta \mathcal{L}=\mathcal{L}^{\text {bef }}-\mathcal{L}^{\text {new }}=\left[I\left(T^{\text {bef }} ; Y\right)-I\left(T^{\text {new }} ; Y\right)\right]+ \\
& \sum_{i=1}^{k}\left[I\left(T^{\text {bef }} ; W_{i}\right)-I\left(T^{\text {new }} ; W_{i}\right)\right]- \\
& \beta^{-1}\left[I\left(T^{\text {bef }} ; X\right)-I\left(T^{\text {new }} ; X\right)\right] \\
& =\Delta I_{\text {content }}+\sum_{i=1}^{k} \Delta I_{\text {contex }}^{i}-\beta^{-1} \Delta I_{\text {compress }},
\end{aligned}
$$

where $T^{\text {bef }}$ and $T^{\text {new }}$ are the categories before and after $x$ is merged. $\Delta I_{\text {content }}$ is the merger cost caused by content information $Y . \Delta I_{\text {context }}^{i}$ is the merger cost caused by the $i$-th visual context $W_{i} . \Delta I_{\text {compress }}^{i}$ is the merger cost by compressing $X$ into its compressed representation $T$. From Eq. (8), we can see that the merge cost $\Delta \mathcal{L}$ can be obtained from the calculation of $\Delta I_{\text {content }}, \Delta I_{\text {context }}^{i}$ and $\Delta I_{\text {compress }}$, respectively. According to (6), we can work out the following equations

$$
\begin{aligned}
& \Delta I_{\text {content }}=I\left(T^{\text {bef }} ; Y\right)-I\left(T^{\text {new }} ; Y\right) \\
& =p(x) \sum_{y} p(y \mid x) \log \frac{p(y \mid x)}{p(y)}+p(t) \sum_{y} p(y \mid t) \log \frac{p(y \mid t)}{p(y)} \\
& -p\left(t^{\prime}\right) \sum_{y} p\left(y \mid t^{\prime}\right) \log \frac{p\left(y \mid t^{\prime}\right)}{p(y)} \\
& =p(x) \sum_{y} p(y \mid x) \log \frac{p(y \mid x)}{p(y)}+p(y) \sum_{y} p(y \mid t) \log \frac{p(y \mid t)}{p(y)} \\
& -\sum_{y} p(x) p(y \mid x) \log \frac{p\left(y \mid t^{\prime}\right)}{p(y)}-\sum_{y} p(t) p(y \mid t) \log \frac{p\left(y \mid t^{\prime}\right)}{p(y)} \\
& =p(x) \sum_{y} p(y \mid x) \log \frac{p(y \mid x)}{p\left(y \mid t^{\prime}\right)}+p(t) \sum_{y} p(y \mid t) \log \frac{p(y \mid t)}{p\left(y \mid t^{\prime}\right)} \\
& =p(x) D_{K L}\left[p(y \mid x) \| p\left(y \mid t^{\prime}\right)\right]+p(t) D_{K L}\left[p(y \mid t) \| p\left(y \mid t^{\prime}\right)\right] \\
& =[p(x)+p(t)] J S_{\Pi}[p(y \mid x), p(y \mid t)] .
\end{aligned}
$$

where $J S_{\Pi}$ is the Jensen-Shannon (JS) divergence [29] to compute the distance of two distributions, and $\Pi=\left\{\pi_{1}, \pi_{2}\right\}=$ $\left\{\frac{p(x)}{p(x)+p(t)}, \frac{p(t)}{p(x)+p(t)}\right\}$. Since $p\left(t^{\prime}\right) \geq 0$ and $J S_{\Pi} \geq 0$, we can get that $\Delta I_{\text {content }} \geq 0$. Similar analysis yields

$$
\Delta I_{\text {compress }}=[p(x)+p(t)] J S_{\Pi}[p(x), p(x \mid t)] .
$$

\section{Relatedness Between Content and Visual Contexts}

Now, we give the calculation of $\Delta I_{\text {context. }}^{i}$. The sequential draw-and-merge procedure is an iterative procedure in essence. We use $T^{\text {mid }}=\left\{t_{1}^{\text {mid }}, t_{2}^{\text {mid }}, \cdots, t_{M}^{\text {mid }}\right\}$ to present temporary partition in each iteration of CMIB conducted by content variable, where $M$ is the number of categories. Similarly, let $W^{l}$ be one partition of multiple auxiliary clusterings $W_{1}, W_{2}, \ldots, W_{k}$, taking values from $W^{l}=$ $\left\{w_{1}^{l}, w_{2}^{l}, \cdots, w_{M}^{l}\right\}$. To measure the relationship between content information and the visual contexts, we should construct the co-occurrence matrix of each pair of them.

As mentioned earlier, there are $m$ data elements in unlabeled image collection $X$, taking values from $\left\{x_{1}, x_{2}, \ldots, x_{m}\right\}$. Let $m_{i}$ be the number of data points allocated into category $t_{i}^{m i d}$; let $m_{j}$ be the number of data points allocated into category $c_{j}^{l}$; let $m_{i j}$ be the number of data points allocated into category $t_{i}^{m i d}$ and $c_{j}^{l}$ at the same time. Thus, the joint co-occurrence distribution of category $T^{\text {mid }}$ and $C^{l}$ can be computed as follows: $p\left(t_{i}^{\text {mid }}\right)=m_{i} / m, p\left(t_{j}^{\text {mid }}\right)=m_{j} / m, p\left(t_{i}^{\text {mid }}, c_{j}^{l}\right)=m_{i j} / m$. Given feature variables and auxiliary clusterings, now we can calculate their mutual information. Thus, the merger cost in Eq. (8) can be obtained now. The pseudo-code of CMIB is given in Algorithm 1. 


\section{E. Theoretical Analysis}

1) Convergence: Now we give Theorem 1.

Theorem 1. The objective function of CMIB algorithm can converge to a stable solution.

Proof. Assuming $X$ has true clustering $W$, we can obtain $I\left(T ; W_{i}\right) \leq I\left(X ; W_{i}\right)$. And since $T$ is a squeezed representation of $X$, so $I(T ; Y) \leq I(X ; Y)$ and $I(X ; T) \leq$ $I(X ; X)$. Therefore, we conclude that the function $(5)$ is upper bounded. In the framework of CMIB, since the single category $\{x\}$ is merged into the category $t^{\text {new }}$ such that $t^{\text {new }}=\arg \min _{t \in T} \Delta \mathcal{L}$, there must be some information loss once $x$ is merged into some new categories, i.e., $\Delta \mathcal{L} \geq 0$. In other words, the "draw-and-merge" optimization approach will increase the function (5). Thus, the objective function of CMIB algorithm can converge into a stable final solution.

2) Complexity: In this part, we analyze the time complexity of CMIB. At step 2, the source data $X$ is partitioned into different categories with random initialization, so this step takes $O(|X|)$, where $|X|$ is the number of data points. In the main loop, the complexity of drawing data point $x$ at step 5 is $O(|X|)$. The computation of the merge cost in step 6 takes $O(M|Y|)$, where $M$ is the number of categories. In the next experimental section, we will show the convergence of the objective function (5). Therefore, the overall time complexity of CMIB is $O(M|X||Y|)$.

\section{EXPERIMENTS}

In this section, we conduct experiments to demonstrate the effectiveness of the proposed CMIB. Specifically, we first verify the performance of CMIB compared with five types of state-of-the-art approaches. Then, we explore the major factors that affect the performance of CMIB.

\section{A. Image data set}

In our experiments, five real-world image data sets including different object categories are employed for evaluation, which are Soccer [30], A-Yahoo [31], Dslr, Webcam and Amazon [32]. The Soccer 1 is collected from 7 soccer teams, containing 40 images per class, total of 280 images. The AYahoo consists of 12 objects which is collected from the Yahoo image search. We select 11 categories in our experiments, with each category containing 100 images. Dslr, Webcam and Amazon data sets consist of 31 types of the object categories, which are the products recorded from different equipment.

\section{B. Data representation}

We exploit the popular bag-of-visual-words (BoVW) model to represent the image collections, which has been widely used in various vision tasks. To represent an image collection, the following four steps should be implemented. 1) Feature detection. Local patches are extracted from each image, and the descriptors (SIFT, Color Attention and TPLBP, respectively)

\footnotetext{
${ }^{1}$ http://lear.inrialpes.fr/people/vandeweijer/data

${ }^{2}$ http://vision.cs.uiuc.edu/attributes/
}

are adopted to represent them. 2) Codebook construction. A visual codebook is built by $k$-means algorithm for each type of features, in which the visual words are all the cluster centers. 3) Mapping descriptors into codebook. All the descriptors are mapped into their corresponding visual words. 4) Counting occurrence. The number of occurrence of the visual words in each image is counted, thus each image can be represented by a vector including occurrence number of visual words.

To construct the content and visual contexts, we utilize three features (SIFT [25], Color Attention [26] and TPLBP [27]) to describe the images, respectively. In this study, we designate one discriminative feature as the content information of the object, while the remaining two features are utilized to generate visual contexts. Specifically, it is easy to differentiate soccer teams by the color of football shirt, so we treat the color feature as the content information for Soccer data. Since the other four data sets consist of the images of products collected from different websites, it is suitable to regard the discriminative shape feature (SIFT) as their content feature.

\section{Comparison methods}

We adopt five types of comparison methods, they are 1) Original information bottleneck. 2) Traditional clustering methods: $k$-means, normalized cuts (NCuts) [33], probabilistic latent semantic analysis (pLSA) [34], and latent dirichlet allocation (LDA) [35]. 3) Ensemble clustering methods: clusterbased similarity partitioning algorithm (CSPA), hyper graph partitioning algorithm (HGPA), meta-clustering algorithm (MCLA) and locally weighted evidence accumulation (LWGP). 4) Multi-view clustering methods: co-regularized multi-view spectral clustering (CRSC) [16], co-training multi-view spectral clustering (CTSC) [15] and robust multi-view spectral clustering (RMSC) [17]. 5) Image clustering methods: local discriminant models and global integration (LDMGI) [36], clustering-by-composition (CC) [37] and ensemble projection (EP) [38]. All source codes of the comparisons were provided by the original authors.

\section{Evaluation metrics}

In this paper, we employ two metrics, normalized mutual information (NMI) and clustering accuracy (AC) [15], to evaluate the performance of different methods.

Given an image $x_{i}$, let $l_{i}$ and $t_{i}$ be the ground truth label and obtained cluster label respectively, so AC is:

$$
A C=\frac{\sum_{i=1}^{n} \delta\left(l_{i}, \operatorname{map}\left(t_{i}\right)\right)}{n},
$$

where $n$ is the total number of images and $\delta\left(l_{i}, \operatorname{map}\left(t_{i}\right)\right)$ is the delta function that equals 1 if $x=y$ and equals 0 otherwise, and $\operatorname{map}\left(t_{i}\right)$ is the optimal mapping function that permutes clustering labels to match the ground truth labels. The optimal mapping can be obtained by using the KuhnMunkres algorithm [39].

Different from AC, NMI is an information theoretic based evaluation metric [18], which is defined as

$$
N M I=\frac{\sum_{h, l} n_{h, l} \log \left(\frac{n \cdot n_{h, l}}{n_{h} n_{l}}\right)}{\sqrt{\left(\sum_{h} n_{h} \log \frac{n_{h}}{n}\right)\left(\sum_{l} n_{l} \log \frac{n_{l}}{n}\right)}},
$$


TABLE I

THE AC (\%) COMPARISON OF CMIB WITH THE ORIGINAL IB AND OTHER FOUR TRADITIONAL CLUSTERING METHODS.

\begin{tabular}{|c|c|c|c|c|c|c|c|c|}
\hline \multirow{2}{*}{ Data sets } & \multicolumn{3}{|c|}{ IB } & \multirow{2}{*}{$k$-means } & \multirow{2}{*}{ NCuts } & \multirow{2}{*}{ pLSA } & \multirow{2}{*}{ LDA } & \multirow{2}{*}{ CMIB } \\
\hline & SIFT & Color Attention & TPLBP & & & & & \\
\hline Soccer & $35.86 \pm 1.9$ & $51.82 \pm 4.4$ & $23.04 \pm 1.3$ & $43.14 \pm 2.2$ & $49.46 \pm 0.2$ & $47.04 \pm 3.9$ & $49.04 \pm 4.0$ & $55.50 \pm \mathbf{3 . 8}$ \\
\hline A-Yahoo & $26.88 \pm 1.1$ & $18.61 \pm 0.7$ & $32.81 \pm 1.2$ & $23.43 \pm 1.3$ & $21.43 \pm 0.2$ & $31.39 \pm 1.4$ & $32.93 \pm 0.9$ & $35.14 \pm 1.4$ \\
\hline Dslr & $42.81 \pm 1.5$ & $34.22 \pm 1.3$ & $39.18 \pm 2.0$ & $32.63 \pm 2.0$ & $31.49 \pm 0.7$ & $30.82 \pm 1.5$ & $33.01 \pm 1.2$ & $\mathbf{4 8 . 4 7} \pm \mathbf{0 . 8}$ \\
\hline Webcam & $38.84 \pm 1.3$ & $28.04 \pm 1.2$ & $35.57 \pm 1.0$ & $20.84 \pm 1.2$ & $31.56 \pm 0.6$ & $29.60 \pm 1.7$ & $28.73 \pm 1.1$ & $41.42 \pm 2.5$ \\
\hline Amazon & $24.48 \pm 0.8$ & $11.92 \pm 0.4$ & $26.65 \pm 1.2$ & $13.33 \pm 0.5$ & $15.08 \pm 0.2$ & $18.85 \pm 1.4$ & $15.86 \pm 1.3$ & $28.25 \pm 1.5$ \\
\hline Average & 33.77 & 28.92 & 31.45 & 26.67 & 29.80 & 31.54 & 31.91 & 41.76 \\
\hline
\end{tabular}

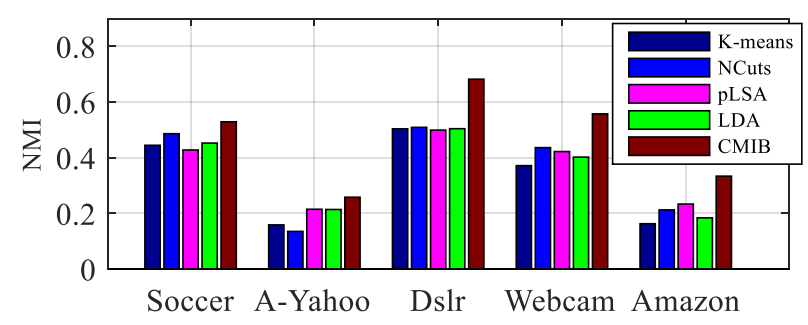

Fig. 4. The comparison of NMI between CMIB and the traditional clustering methods.

where $n$ is the number of images, $n_{l}$ and $n_{h}$ denoting the number of images in category $l$ and cluster $h$, respectively, and $n_{h, l}$ is the number of images in both category $h$ and cluster $l$. The higher the NMI score is the better the clustering quality can be obtained.

\section{E. Experimental results}

In this part, extensive experiments are conducted to demonstrate the effectiveness of CMIB compared with five types of comparison methods.

1) Comparison with original IB method: We conduct experiments to verify the performance of CMIB compared with the original IB method, which can only process the single content feature. From Table II we can get the following observations. Firstly, the clustering results (AC) of the IB algorithm on the three cues are different from each other. This demonstrates that single feature is not discriminative and stable enough for different data sets. Thus, it is unwise to only consider content feature on the mission of unsupervised object categorization. Secondly, by incorporating the visual contexts, the proposed CMIB method performs better than the IB algorithm clearly. For instance, as shown in CMIB column in Table I. the performance improvements (average AC) of CMIB method on the five image data sets are $7.99 \%, 12.84 \%$, $10.31 \%$ respectively compared with IB on the three cues. We obtain that the CMIB outperforms the IB significantly by dealing with content and visual contexts simultaneously.

2) Comparison with traditional clustering methods: In this part, the experiments are conducted to compare CMIB algorithm with other four traditional clustering approaches. In particular, the $k$-means is one of most versatile clustering method, which is to find cluster centers that minimize the intra-class variance. The NCuts [33] is a graph based partition clustering method, which measures both the total dissimilarity between the different groups as well as the total similarity

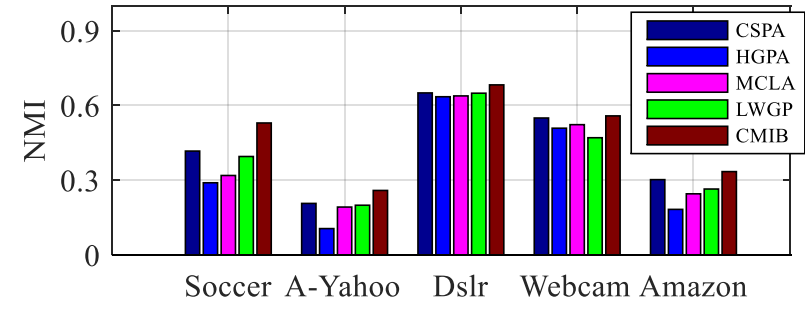

Fig. 5. The comparison of NMI between CMIB and the ensemble clustering methods.

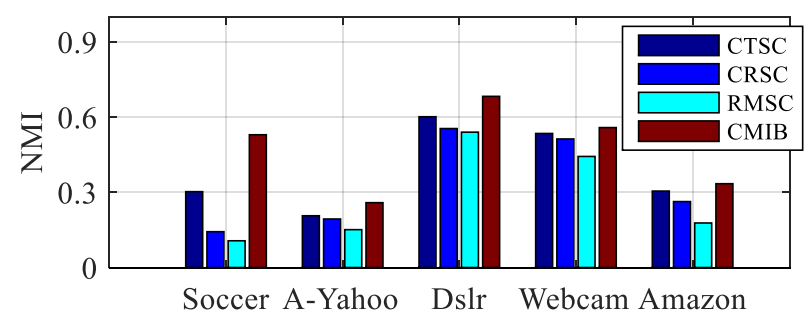

Fig. 6. The comparison of NMI between CMIB and the multi-view clustering methods.

within the groups. The pLSA [34] and LDA [35] are two statistical techniques for the analysis of co-occurrence data. From Table 1 and Fig. 4, we observe that our CMIB method can obtain more promising results compared with the other traditional clustering methods, which is mainly caused by the visual contexts used in our CMIB method.

3) Comparison with ensemble clustering methods: We further conduct the comparative experiments with ensemble clustering methods (CSPA, HGPA, MCLA [18] and LWGP [19]) to demonstrate the effectiveness of CMIB. Ensemble clustering methods combine multiple clusterings into a single consolidated partition, which is a consensus of multiple base clusterings. We utilize the original IB to construct 30 base clusterings for the above ensemble clustering methods, in which each feature generate 10 clusterings, respectively. From Table $\Pi$ and Fig. 5, the CMIB outperforms all the four ensemble clustering methods on the five data sets. This is mainly because ensemble clustering methods usually limit the final results to the quality of existing base clusterings. The proposed CMIB method can deal with original feature (content feature) and visual contexts (basic clusterings) simultaneously and relieve the overreliance of ensemble clustering methods on auxiliary clusterings.

4) Comparison with multi-view clustering methods: To further demonstrate the performance of CMIB, we compare 
TABLE II

THE AC (\%) COMPARISON OF CMIB WITH ENSEMBLE AND MULTI-VIEW CLUSTERING METHODS.

\begin{tabular}{c|cccc|ccc|c}
\hline \multirow{2}{*}{ Data sets } & \multicolumn{4}{|c|}{ Ensemble clustering } & \multicolumn{3}{c|}{ Multi-view clustering } & \multirow{2}{*}{ CMIB } \\
\cline { 2 - 9 } & CSPA & HGPA & MCLA & LWGP & CTSC & CRSC & RMSC & \\
\hline Soccer & $53.93 \pm 0.1$ & $39.89 \pm 6.0$ & $47.11 \pm 0.3$ & $50.69 \pm 2.3$ & $38.39 \pm 3.7$ & $31.46 \pm 1.9$ & $27.04 \pm 1.8$ & $\mathbf{5 5 . 5 0} \pm \mathbf{3 . 8}$ \\
A-Yahoo & $32.79 \pm 0.3$ & $20.75 \pm 1.4$ & $32.17 \pm 0.6$ & $32.46 \pm 1.2$ & $31.43 \pm 1.1$ & $29.00 \pm 0.6$ & $25.20 \pm 0.4$ & $\mathbf{3 5 . 1 4} \pm \mathbf{1 . 4}$ \\
Dslr & $45.58 \pm 0.5$ & $39.38 \pm 1.5$ & $42.25 \pm 0.4$ & $41.74 \pm 2.1$ & $41.29 \pm 1.5$ & $36.08 \pm 1.3$ & $35.92 \pm 1.5$ & $\mathbf{4 8 . 4 7} \pm \mathbf{0 . 8}$ \\
Webcam & $40.75 \pm 0.1$ & $33.19 \pm 2.1$ & $37.42 \pm 0.7$ & $39.85 \pm 2.2$ & $38.25 \pm 2.0$ & $36.31 \pm 0.8$ & $28.74 \pm 1.1$ & $\mathbf{4 1 . 4 2} \pm \mathbf{2 . 5}$ \\
Amazon & $25.19 \pm 0.6$ & $14.67 \pm 0.7$ & $22.85 \pm 0.4$ & $23.37 \pm 1.5$ & $26.08 \pm 1.0$ & $21.32 \pm 0.6$ & $15.43 \pm 0.5$ & $\mathbf{2 8 . 2 5} \pm \mathbf{1 . 5}$ \\
\hline Average & 39.65 & 29.58 & 36.36 & 37.62 & 35.09 & 30.83 & 26.47 & $\mathbf{4 1 . 7 6}$ \\
\hline
\end{tabular}

it with other three multi-view clustering methods, which are CTSC [15], CRSC [16] and RMSC [17]. In this experiment, we treat each feature representation (SIFT, Color Attention and TPLBP) as one input view of the multi-view clustering methods. From Table $[\mathrm{II}$ and Fig. 6, we can get that the performances of CMIB are better than the multi-view clustering methods significantly.

5) Comparison with image clustering methods: For the comparison with state-of-the-art unsupervised object categorization methods, we adopt local discriminant models and global integration (LDMGI) [36], clustering-by-composition (CC) [37] and ensemble projection (EP) [38] as baselines. LDMGI learns a new Laplacian matrix by using both manifold structure and local discriminant information. CC composes similar images easily from each other, and non-similar images will be a lot harder to compose from each other. EP learns a new feature representation by capturing the information of each image as well as the relatedness across images. As shown in Table III] the average AC values of CMIB algorithm on the five data sets obtains $9.38 \%, 2.9 \%$, and $9.64 \%$ improvement respectively compared the other three baselines. We get same observations in terms of NMI in Fig. 7

\section{F. Explanation of impact factors}

1) Parameter analysis: In CMIB, $\beta$ strikes a balance between information preservation and data compression. Thus, we conduct experiment to investigate the impact of $\beta$ on the performance of CMIB. Specifically, we vary the values of $\beta$ from the space $\{1,5,10,15,20,40,60,80,100,140\}$. From Fig. 8, we can get the following observations: First, when $\beta \rightarrow 0$, CMIB performs poorly since it only interested in the compression from source images $X$ to its compressed representation $T$, i.e., the object category in this study. When increasing the value of $\beta$, CMIB performs much better because it strikes a balance between the data compression and information preservation. We set the $\beta$ to 40 on all the data sets in this study.

2) Convergence analysis: Fig. 9 shows the iteration number of the CMIB algorithm on the five data sets. It can be observed that the values of function (5) increase monotonically and can reach convergence rapidly in a limited number of iterations.

\section{CONCLUSIONS}

We propose a novel contextual multivariate information bottleneck (CMIB) approach, which aims to discover the object category in unlabeled images by considering content feature
TABLE III

THE AC (\%) COMPARISON OF CMIB WITH STATE-OF-THE-ART IMAGE CLUSTERING METHODS.

\begin{tabular}{c|cccc}
\hline Data sets & LDMGI & CC & EP & CMIB \\
\hline Soccer & $43.21 \pm 0.5$ & $47.75 \pm 2.2$ & $48.21 \pm 0.4$ & $\mathbf{5 5 . 5 0} \pm \mathbf{3 . 8}$ \\
A-Yahoo & $28.11 \pm 1.1$ & $32.27 \pm 0.6$ & $30.87 \pm 1.5$ & $\mathbf{3 5 . 1 4} \pm \mathbf{1 . 4}$ \\
Dslr & $37.51 \pm 1.6$ & $47.27 \pm 1.7$ & $36.22 \pm 1.6$ & $\mathbf{4 8 . 4 7} \pm \mathbf{0 . 8}$ \\
Webcam & $33.53 \pm 1.0$ & $40.97 \pm 0.8$ & $24.47 \pm 0.6$ & $\mathbf{4 1 . 4 2} \pm \mathbf{2 . 5}$ \\
Amazon & $19.56 \pm 0.7$ & $26.04 \pm 1.0$ & $20.81 \pm 0.5$ & $\mathbf{2 8 . 2 5} \pm \mathbf{1 . 5}$ \\
\hline Average & 32.38 & 38.86 & 32.12 & $\mathbf{4 1 . 7 6}$ \\
\hline
\end{tabular}

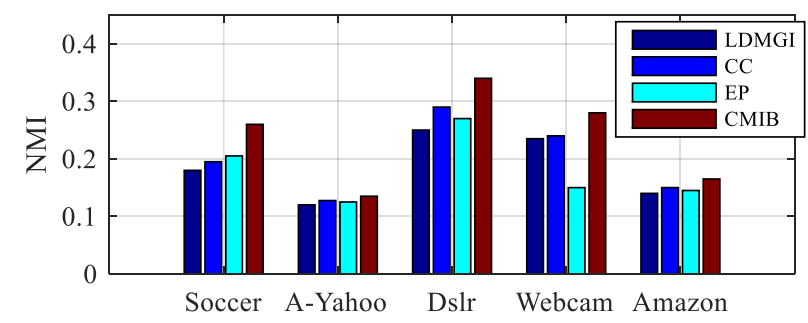

Fig. 7. The comparison of NMI between CMIB and the image clustering methods.

and their visual contexts simultaneously. Differently, we focus on totally unsupervised setting. CMIB treats object category discovery as a procedure of data compression, in which both content and contextual information can be preserved maximally. Specifically, CMIB utilizes two Bayesian networks to characterize the relationships between data compression and information preservation. We present extensive experiments showing that the performance of our CMIB method is superior to other existing state-of-the-art baselines. In our future research, we will investigate more visual contexts and test the proposed method on more realistic applications.

\section{REFERENCES}

[1] Y. Park and I. S. Kweon, "Ambiguous surface defect image classification of amoled displays in smartphones," IEEE Transactions on Industrial Informatics, vol. 12, no. 2, pp. 597-607, 2016.

[2] J. Yang, B. Jiang, B. Li, K. Tian, and Z. Lv, "A fast image retrieval method designed for network big data," IEEE Transactions on Industrial Informatics, vol. 13, no. 5, pp. 2350-2359, 2017.

[3] L. Zhao, Z. He, W. Cao, and D. Zhao, "Real-time moving object segmentation and classification from hevc compressed surveillance video," IEEE Transactions on Circuits and Systems for Video Technology, vol. PP, no. 99, pp. 1-1, 2017 (Early Access).

[4] S. Kumar and M. Hebert, "A hierarchical field framework for unified context-based classification," in International Conference on Computer Vision, 2005, pp. 1284-1291.

[5] A. Rabinovich, A. Vedaldi, C. Galleguillos, E. Wiewiora, and S. Belongie, "Objects in context," in International Conference on Computer Vision, 2007, pp. 1-8. 


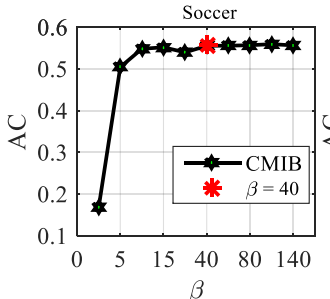

$\beta$

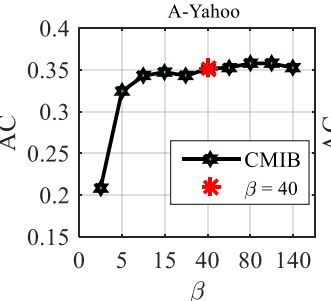

$\beta$

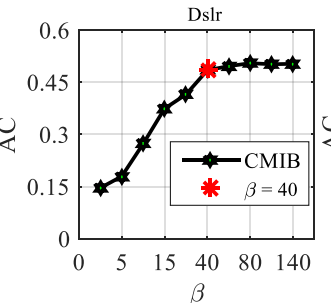

$\beta$
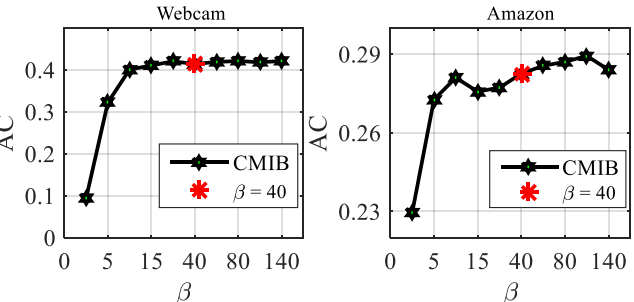

Fig. 8. The AC values of CMIB on the five data sets with different trade-off parameters.
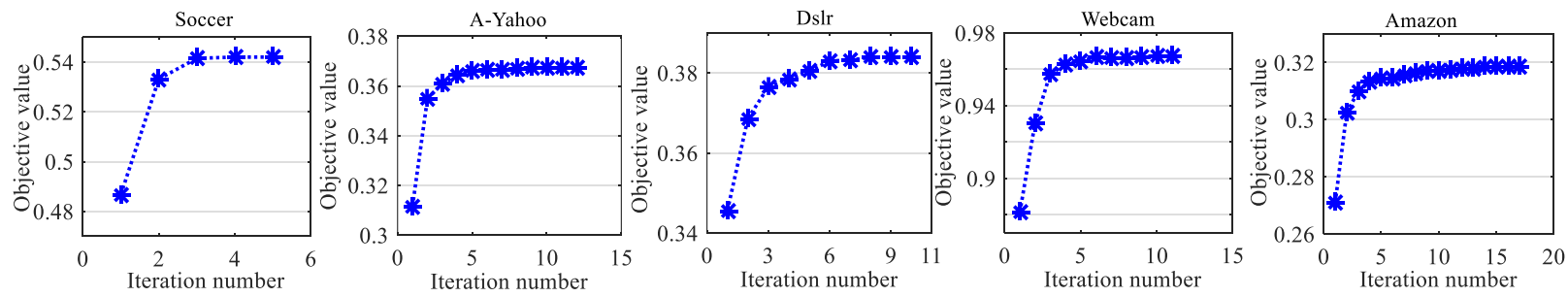

Fig. 9. The iterations of CMIB on the five data sets.

[6] M. J. Choi, J. J. Lim, A. Torralba, and A. S. Willsky, "Exploiting hierarchical context on a large database of object categories," in IEEE Conference on Computer Vision and Pattern Recognition, 2010, pp. 129136.

[7] X. Song, S. Jiang, and L. Herranz, "Joint multi-feature spatial context for scene recognition on the semantic manifold," in IEEE Conference on Computer Vision and Pattern Recognition, 2015, pp. 1312-1320.

[8] L. Yang, K. Tang, J. Yang, and L. J. Li, "Dense captioning with joint inference and visual context," in IEEE Conference on Computer Vision and Pattern Recognition, 2017, pp. 1978-1987.

[9] J. Yuan and Y. Wu, "Context-aware clustering," in IEEE Conference on Computer Vision and Pattern Recognition, 2008, pp. 1-8.

[10] H. Wang, J. Yuan, and Y. Tan, "Combining feature context and spatial context for image pattern discovery," in International Conference on Data Mining, 2011, pp. 764-773.

[11] V. Nguyen, D. Phung, X. Nguyen, and H. H. Bui, "Bayesian nonparametric multilevel clustering with group-level contexts," in International Conference on Machine Learning, 2014, pp. 288-296.

[12] D. Harwath, A. Torralba, and J. Glass, "Unsupervised learning of spoken language with visual context," in Advances in Neural Information Processing Systems, 2016, pp. 1858-1866.

[13] J. Wang, Z. Chen, and Y. Wu, "Action recognition with multiscale spatiotemporal contexts," in IEEE Conference on Computer Vision and Pattern Recognition, 2011, pp. 3185-3192.

[14] S. Jones and L. Shao, "Unsupervised spectral dual assignment clustering of human actions in context," in IEEE Conference on Computer Vision and Pattern Recognition, 2014, pp. 604-611.

[15] A. Kumar and H. Daume, "A co-training approach for multi-view spectral clustering," in International Conference on Machine Learning, 2011, pp. 393-400.

[16] A. Kumar, P. Rai, and H. Daume, "Co-regularized multi-view spectral clustering," in Advances in Neural Information Processing Systems, 2011, pp. 1413-1421.

[17] R. Xia, Y. Pan, L. Du, and J. Yin, "Robust multi-view spectral clustering via low-rank and sparse decomposition," in AAAI Conference on Artificial Intelligence, 2014, pp. 2149-2155.

[18] A. Strehl and J. Ghosh, "Cluster ensembles-a knowledge reuse framework for combining multiple partitions," Journal of Machine Learning Research, vol. 3, pp. 583-617, 2003.

[19] D. Huang, C. D. Wang, and J. H. Lai, "Locally weighted ensemble clustering," IEEE Transactions on Cybernetics, vol. 48, no. 5, pp. 14601473, 2018.

[20] N. Tishby, F. C. Pereira, and W. Bialek, "The information bottleneck method," in Annual Allerton Conference on Communnication, Control and Computing, 1999, pp. 368-377.

[21] N. Slonim, N. Friedman, and N. Tishby, "Multivariate information bottleneck," Neural Computation, vol. 18, no. 8, pp. 1739-1789, 2006.
[22] Y. J. Lee and K. Grauman, "Object-graphs for context-aware visual category discovery," IEEE Transactions on Pattern Analysis and Machine Intelligence, vol. 34, no. 2, pp. 346-358, 2012.

[23] H. Wang, A. Kläser, C. Schmid, and C. Liu, "Dense trajectories and motion boundary descriptors for action recognition," International Journal of Computer Vision, vol. 103, no. 1, pp. 60-79, 2013.

[24] M. Van, S. S. Ge, and D. Ceglarek, "Fault estimation and accommodation for virtual sensor bias fault in image-based visual servoing using particle filter," IEEE Transactions on Industrial Informatics, vol. 14 no. 4, pp. 1312-1322, 2018.

[25] D. G. Lowe, "Distinctive image features from scale-invariant keypoints," International Journal of Computer Vision, vol. 60, no. 2, pp. 91-110, 2004.

[26] F. S. Khan, J. D. Weijer, and M. Vanrell, “Top-down color attention for object recognition," in International Conference on Computer Vision, 2009, pp. 979-986.

[27] L. Wolf, T. Hassner, and Y. Taigman, "Descriptor based methods in the wild," in European Conference on Computer Vision, 2008.

[28] J. Philbin, O. Chum, J. Sivic, and A. Zisserman, "Object retrieval with large vocabularies and fast spatial matching," in IEEE Conference on Computer Vision and Pattern Recognition, 2007, pp. 1-8.

[29] T. M. Cover and J. A. Thomas, Elements of Information Theory, 1991.

[30] J. V. D. Weijer and C. Schmid, "Coloring local feature extraction," in European Conference on Computer Vision, 2006, pp. 334-348.

[31] A. Farhadi, I. Endres, D. Hoiem, and D. Forsyth, "Describing objects by their attributes," in IEEE Conference on Computer Vision and Pattern Recognition, 2009, pp. 1778-1785.

[32] K. Saenko, B. Kulis, M. Fritz, and T. Darrell, "Adapting visual category models to new domains," in European Conference on Computer Vision, 2010, pp. 213-226

[33] J. Shi and J. Malik, "Normalized cuts and image segmentation," IEEE Transactions on Pattern Analysis and Machine Intelligence, vol. 22, no. 8, pp. 888-905, 2000.

[34] T. Hofmann, "Probabilistic latent semantic analysis," in Conference on Uncertainty in Artificial Intelligence, 1999, pp. 289-296.

[35] D. M. Blei, A. Y. Ng, and M. I. Jordan, "Latent dirichlet allocation," Journal of Machine Learning Research, vol. 3, pp. 993-1022, 2003.

[36] Y. Yang, D. Xu, F. Nie, S. Yan, and Y. Zhuang, "Image clustering using local discriminant models and global integration," IEEE Transactions on Image Processing, vol. 19, no. 10, pp. 2761-2773, 2010.

[37] A. Faktor and M. Irani, "Clustering by composition - unsupervised discovery of image categories," IEEE Transactions on Pattern Analysis and Machine Intelligence, vol. 36, no. 6, pp. 1092-1106, 2014.

[38] D. Dai and L. J. V. Gool, "Unsupervised high-level feature learning by ensemble projection for semi-supervised image classification and image clustering," CoRR, vol. abs/1602.00955, 2016.

[39] C. H. Papadimitriou and K. Steiglitz, Combinatorial optimization: algorithms and complexity. Dover Publications, 1998. 